\title{
Tamoxifen therapy benefit for patients with 70-gene signature high and low risk
}

\author{
Laura J. van 't Veer' ${ }^{1}$ Christina Yau', ${ }^{2,3}$ Nancy Y. Yu' ${ }^{5}$ Christopher C. Benz ${ }^{3,4}$. \\ Bo Nordenskjöld ${ }^{6} \cdot$ Tommy Fornander $^{7} \cdot$ Olle Stål $^{6}$ - Laura J. Esserman ${ }^{2}$. \\ Linda Sofie Lindström ${ }^{5}$
}

Received: 26 July 2017/ Accepted: 28 July 2017/Published online: 4 August 2017

(c) The Author(s) 2017. This article is an open access publication

\begin{abstract}
Background Breast cancer molecular prognostic tools that predict recurrence risk have mainly been established on endocrine-treated patients and thus are not optimal for the evaluation of benefit from endocrine therapy. The Stockholm tamoxifen (STO-3) trial which randomized postmenopausal node-negative patients to 2-year tamoxifen (followed by an optional randomization for an additional 3-year tamoxifen vs nil), versus no adjuvant treatment,
\end{abstract}

Electronic supplementary material The online version of this article (doi:10.1007/s10549-017-4428-9) contains supplementary material, which is available to authorized users.

Laura J. van 't Veer

laura.vantveer@ucsf.edu

Linda Sofie Lindström

linda.lindstrom@ki.se

1 Department of Laboratory Medicine, University of California San Francisco, 2340 Sutter Street, San Francisco, CA 94115, USA

2 Department of Surgery, University of California San Francisco, 1600 Divisadero Street, San Francisco, CA 94115, USA

3 Buck Institute for Research on Aging, 8001 Redwood Boulevard, Novato, CA 94945, USA

4 Department of Medicine, University of California San Francisco, 1600 Divisadero Street, San Francisco, CA 94115, USA

5 Department of Biosciences and Nutrition, Karolinska Institutet, Hälsovägen 7, 14183 Stockholm, Sweden

6 Department of Clinical and Experimental Medicine and Department of Oncology, Linköping University, Sandbäcksgatan 7, 58183 Linköping, Sweden

7 Department of Oncology-Pathology, Karolinska Institutet, Z1:00, 17176 Stockholm, Sweden provides a unique opportunity to evaluate long-term 20-year benefit of endocrine therapy within prognostic risk classes of the 70-gene prognosis signature that was developed on adjuvantly untreated patients.

Methods We assessed by Kaplan-Meier analysis 20-year breast cancer-specific survival (BCSS) and 10-year distant metastasis-free survival (DMFS) for 538 estrogen receptor (ER)-positive, STO-3 trial patients with retrospectively ascertained 70-gene prognosis classification. Multivariable analysis of long-term (20 years) BCSS by STO- 3 trial arm in the 70-gene high-risk and low-risk subgroups was performed using Cox proportional hazard modeling adjusting for classical patient and tumor characteristics.

Results Tamoxifen-treated, 70-gene low- and high-risk patients had 20-year BCSS of 90 and $83 \%$, as compared to 80 and $65 \%$ for untreated patients, respectively (log-rank $p<0.0001)$. Notably, there is equivalent tamoxifen benefit in both high (HR $0.42(0.21-0.86), p=0.018)$ and low (HR $0.46(0.25-0.85), p=0.013) 70$-gene risk categories even after adjusting for clinico-pathological factors for BCSS. Limited tamoxifen exposure as given in the STO-3 trial provides persistent benefit for 10-15 years after diagnosis in a time-varying analysis. 10-year DMFS was 93 and $85 \%$ for low- and high-risk tamoxifen-treated, versus 83 and $70 \%$ for low- and high-risk untreated patients, respectively (log-rank $p<0.0001)$.

Conclusions Patients with ER-positive breast cancer, regardless of high or low 70-gene risk classification, receive significant survival benefit lasting over 10 years from adjuvant tamoxifen therapy, even when given for a relatively short duration.

Keywords 70-gene signature - Tamoxifen benefit . Endocrine therapy $\cdot$ Breast cancer $\cdot$ Long-term survival 


\section{Introduction}

Breast cancer is a diverse disease both in the sense of the metastatic potential of the primary tumor as well as time for metastasis to occur. The biological factors influencing the long-term risk of fatal breast cancer are unknown. It is, however, known that patients with newly diagnosed hormone receptor (HR)-positive breast cancer (ER- and/or progesterone (PR)-positive disease) have a continuous long-term risk for fatal breast cancer progression relative to stage-matched patients with newly diagnosed HR-negative cancer [1]. Endocrine therapy remains the cornerstone in management of HR-positive breast cancer since adjuvant use of either tamoxifen or aromatase inhibitors significantly improves the long-term survival of patients with either localized or regional HR-positive breast cancer $[2,3]$. Survival benefit of adjuvant endocrine therapy has been shown to be independent of patient age, menopausal status, quantitative ER expression, nodal status, tumor size, grade, and proliferation rate [3, 4], and almost all newly diagnosed HR-positive breast cancers are nowadays treated with endocrine therapy. Nonetheless, this survival benefit varies markedly among similarly staged patients since up to half of all HR-positive patients receive little or no benefit from adjuvant endocrine therapy [3, 5], presumably due to breast cancer inter-tumor heterogeneity associated with endocrine resistance.

Gene expression signatures have helped us understand the inter-tumor heterogeneity between breast cancer tumors $[6,7]$, separating tumors into subgroups with different underlying biology, prognosis, and treatment benefit [8-10]. Unlike many other gene expression signatures, the 70-gene prognosis signature was developed on a systemically untreated patient cohort [11], which makes the signature suitable to evaluate treatment benefit.

Given the pressing need to understand more about longterm breast cancer survival and endocrine therapy benefit, we evaluated long-term endocrine therapy benefit in women with 70-gene low- and high-risk prognosis signatures using a large Swedish clinical trial (STO-3) having complete long-term (20 years) follow-up of patients randomized to receive adjuvant tamoxifen versus not.

\section{Methods}

\section{The Stockholm tamoxifen (STO-3) trial}

The Stockholm Tamoxifen (STO-3) trial enrolled postmenopausal patients with lymph node-negative breast cancers with tumors less than or equal to $30 \mathrm{~mm}$ in diameter, randomized to 2 years of adjuvant tamoxifen
(40 mg daily) versus no adjuvant treatment. Patients in the tamoxifen arm, who were relapse free after 2 years of tamoxifen and who re-consented, were further randomized to 3 additional years of tamoxifen or none. From the original randomized trial cohort, 808 patients had formalinfixed paraffin-embedded (FFPE) tissue blocks of primary breast cancer tumor available for molecular analyses, and of these, 81 patients were excluded because there was insufficient invasive tumor present for analysis. The remaining 727-patient subset with FFPE material available is well balanced to the original STO-3 cohort with regard to tumor characteristics, such as tumor size, ER status, and treatment arm assignment [12]. All patients included in the STO-3 randomized trial have detailed patient and clinical information. This study followed REMARK criteria [13].

In Sweden, all residents have a unique national registration number, which enables automatic linkage of various records of personal information from Regional and National registers of high validity and essentially complete coverage. Death due to breast cancer was assessed from the Swedish National Cause-of-Death Register with a reported accuracy of more than 96\% from January 1, 1961 and onwards $[14,15]$. The information on cause of death is from death certificates filled out by the treating physicians. Furthermore, information on contralateral breast cancer was assessed from the Swedish National Cancer Registry. Cancer registration has a legal basis in Sweden, and the Swedish Cancer Registry has a breast cancer coverage of more than $96 \%$ in validation studies [16]. Finally, information on distant metastasis was assessed from the Stockholm Breast Cancer Registry, a population-based registry held by the Regional Cancer Centre in Stockholm. The Breast Cancer Registry carries information on all breast cancer diagnoses in the Swedish counties of Stockholm and Gotland since 1976 as well as follow-up information including local relapse and distant metastasis.

The STO-3 trial was approved by the ethical committee at Karolinska Institutet, and participants provided oral consent.

\section{0-gene signature assignments}

Gene expression data were independently generated using custom-designed arrays, Agilent Technologies (CA, USA), containing approximately $32.1 \mathrm{~K}$ probes, representing approximately $21.5 \mathrm{~K}$ unique genes from FFPE breast cancer tumor tissue. Approximately 90\% (or 652 of 727 breast cancer tumors) passed the RNA quality check (according to the diagnostic quality model) and were used in the analysis. The 70-gene (MammaPrint) signature was performed according to standard protocols as previously described, including the use of 465 normalization genes and over 250 probes for hybridization and printing quality 
control. Patient tumor samples were classified into high or low risk by the 0.00 threshold in the MammaPrint index (high up to and low above 0.00 index, respectively). The subgroup of ultralow tumors is defined by MammaPrint index $>+0.355$ [17-19].

\section{ER, PR, HER2, and Ki-67 immunohistochemistry}

538 of the 652 tumors available for 70-gene evaluation were ER positive. Immunohistochemistry (IHC) was retrospectively done for ER, progesterone receptor [PR], human epidermal growth factor receptor 2 [HER2], and Ki-67 using DAKO Link48 Autostainer at the University of California Davis Medical Center (UCDMC). The antibodies used were ER (SP1; Spring Bioscience M301), PR (PgR 636; DAKO IR068), HER2 (HercepTest; DAKO SK001), and Ki67 (MIB-

1; DAKO M7240), with EnVision + detection, following standard recommended procedures and with per-run positive controls assessed by quantitative image analysis to ensure consistent run-to-run staining intensity [19].

\section{Tumor grade}

Tumor grade according to the Nottingham system was retrospectively assessed by one pathologist [12].

\section{Statistical methods}

\section{Survival analyses}

The outcome of interest was death due to breast cancer, and analyses of long-term breast cancer-specific survival (20 year) by the 70 -gene risk classification (high and low risk) were performed in patients with ER-positive tumors. Patient follow-up started at the date of primary breast cancer diagnosis and ended at the date of death, contralateral breast cancer diagnosis, emigration from Sweden (only five women emigrated in total), or end of study follow-up (December 31, 2012).

For comparison with previous studies, we also performed 10-year analysis of distant metastasis-free survival. However, information on metastasis is less complete as compared to information on death. In our study, approximately $2 \%$, i.e., 14 patients out of 727 patients, died from breast cancer but have missing information on metastasis. In patients with ER-positive disease and available gene expression information (538 patients) as included in this study, 11 patients who died from breast cancer had missing information on metastasis. For these 11 patients, date of death was used instead of the date of metastasis.

Kaplan-Meier analyses were performed by STO-3 trial arm and 70-gene risk classification. The significance was assessed using the log-rank test.
Multivariable analysis by the 70-gene risk classification was performed using Cox proportional hazard modeling adjusting for classical patient and tumor characteristics (age and calendar period of diagnosis, progesterone receptor status, HER2 status, Ki-67 status, tumor grade, and tumor size). Multivariable analysis for the ultralow 70-gene risk group by trial arm was not performed due to low sample size.

Flexible parametric survival models were used to estimate hazard ratios over time since diagnosis. Breast cancer-specific death rates were modeled through flexible parametric survival models using a restricted cubic spline function for the baseline mortality rate $[20,21]$. Time-dependent multivariable analysis was performed for 1-, 5-, $10-, 15-$, and 20-year follow-up time points, adjusting for the same patient and tumor characteristics as listed above. A spline with three degrees of freedom was used to estimate the hazard ratios. For the time-dependent covariate (tamoxifen trial arm), we used a second spline function with one degree of freedom to model the interactions between the covariate and time. The stpm 2 function in Stata version 14.2 was used for the modeling and the analyses [20].

The proportional hazard assumption for the main exposure variable (70-gene risk classification) was assessed by including a time-dependent covariate in the model. No significant deviation was noted. Data preparation and analysis were done using SAS version 9.4, Stata version 14.2, and $\mathrm{R}$ version 3.4.0.

\section{Results}

Patients in the STO trial with ER-positive breast cancer disease and available 70-gene expression signature data, 538 patients in total, were included in our analysis. In Table 1, patient and tumor characteristics by the 70-gene risk classification (high versus low risk) are presented. Of 167 patients with tumors classified as being high risk (167/ $538,31 \%$ ), $54 \%$ of the tumors were PR positive, $14 \%$ were HER 2 positive, $41 \%$ had Ki-67 greater than 15 , and $5.5 \%$ of tumors were grade 1 . Of the 371 patients with tumors classified as being low risk (371/538, 69\%), $76 \%$ of the tumors were PR positive, no tumors were HER2 positive, $15 \%$ had Ki-67 greater than 15 , and $29 \%$ of tumors were grade 1.

\section{Survival analysis}

\section{Univariate survival analysis}

Kaplan-Meier survival graphs for patients with and without tamoxifen are shown per trial arm and by the standard 
Table 1 Patient and tumor characteristics by 70 -gene risk classification

\begin{tabular}{|c|c|c|c|c|c|}
\hline & \multicolumn{5}{|c|}{ STO-3 trial } \\
\hline & \multicolumn{2}{|c|}{ 70-Gene high risk } & \multicolumn{2}{|c|}{ 70-Gene low risk } & \multirow{2}{*}{$\begin{array}{l}\text { Total number } \\
\text { of patients }\end{array}$} \\
\hline & Number & Percent & Number & Percent & \\
\hline \multicolumn{6}{|l|}{ STO-3 trial arm } \\
\hline Tamoxifen-treated arm & 82 & 49.1 & 199 & 53.6 & 281 \\
\hline Untreated arm & 85 & 50.9 & 172 & 46.4 & 257 \\
\hline \multicolumn{6}{|l|}{ Patient characteristics } \\
\hline \multicolumn{6}{|l|}{ Calendar period of primary diagnosis } \\
\hline 1976-1984 & 92 & 55.1 & 182 & 49.1 & 274 \\
\hline 1985-1990 & 75 & 44.9 & 189 & 50.9 & 264 \\
\hline \multicolumn{6}{|l|}{ Age at primary diagnosis (years) } \\
\hline $45-54$ & 22 & 13.2 & 30 & 8.1 & 52 \\
\hline $55-64$ & 81 & 48.5 & 184 & 49.6 & 265 \\
\hline $65-74$ & 64 & 38.3 & 157 & 42.3 & 221 \\
\hline \multicolumn{6}{|l|}{ Primary tumor characteristics } \\
\hline \multicolumn{6}{|l|}{ Type of surgery } \\
\hline Breast-conserving surgery and RT & 28 & 16.8 & 93 & 25.1 & 121 \\
\hline Mastectomy & 139 & 83.2 & 278 & 74.9 & 417 \\
\hline \multicolumn{6}{|l|}{ Progesterone receptor status } \\
\hline Positive & 89 & 53.9 & 278 & 76.4 & 367 \\
\hline Negative & 76 & 46.1 & 86 & 23.6 & 162 \\
\hline Unknown & 2 & - & 7 & - & 9 \\
\hline \multicolumn{6}{|l|}{ HER2 status ${ }^{\mathrm{a}}$} \\
\hline Positive & 24 & 14.4 & 0 & 0 & 24 \\
\hline Negative & 143 & 85.6 & 370 & 100 & 513 \\
\hline Unknown & 0 & - & 1 & - & 1 \\
\hline \multicolumn{6}{|l|}{ Ki-67 status ${ }^{b}$} \\
\hline Positive & 66 & 41.3 & 51 & 14.5 & 117 \\
\hline Negative & 94 & 58.7 & 301 & 85.5 & 395 \\
\hline Unknown & 7 & - & 19 & - & 26 \\
\hline \multicolumn{6}{|l|}{ Tumor grade } \\
\hline 1 & 9 & 5.5 & 107 & 29.2 & 116 \\
\hline 2 & 91 & 55.5 & 247 & 67.5 & 338 \\
\hline 3 & 64 & 39.0 & 12 & 3.3 & 76 \\
\hline Unknown & 3 & - & 5 & - & 8 \\
\hline \multicolumn{6}{|l|}{ Tumor size } \\
\hline $\mathrm{pT}<20 \mathrm{~mm}$ & 122 & 74.4 & 315 & 85.6 & 437 \\
\hline $\mathrm{pT} \geq 20 \mathrm{~mm}$ & 42 & 25.6 & 53 & 14.4 & 95 \\
\hline Unknown & 3 & - & 3 & - & 6 \\
\hline
\end{tabular}

${ }^{\text {a }}$ HER2 positive defined as $3+$ by immunohistochemistry

b Ki-67 cut-off for positivity at $15 \%$

70-gene high- and low-risk groups in Fig. 1S. A statistically significant difference in long-term (20 year) breast cancer-specific survival by STO-3 trial arm and 70-gene classification was observed (log-rank $p<0.0001)$. For the 70 -gene low-risk group, the 20-year breast cancer-specific survival with and without tamoxifen treatment was $90 \%$ (95\% CI $84-94 \%)$ and $80.0 \%$ (95\% CI $72-86 \%)$, respectively. For the 70-gene high-risk group, the 20-year breast cancer-specific survival with and without tamoxifen treatment was $83 \%(95 \%$ CI $72-90 \%)$ and $65 \%(95 \% \mathrm{CI}$ 53-75\%), respectively.

The benefit of tamoxifen within each 70-gene risk group is shown in Fig. 1a and b, respectively. A statistically significant difference in long-term (20 year) breast cancer- 
Fig. 1 Kaplan-Meier analysis of breast cancer-specific survival by 70 -gene risk classification and trial arm (tamoxifen treated versus untreated). The $p$ value is based on the log-rank test, and numbers at risk are shown underneath the graph. a 70-gene high risk by trial arm (with and without tamoxifen). b 70-gene low risk by trial arm (with and without tamoxifen)
A

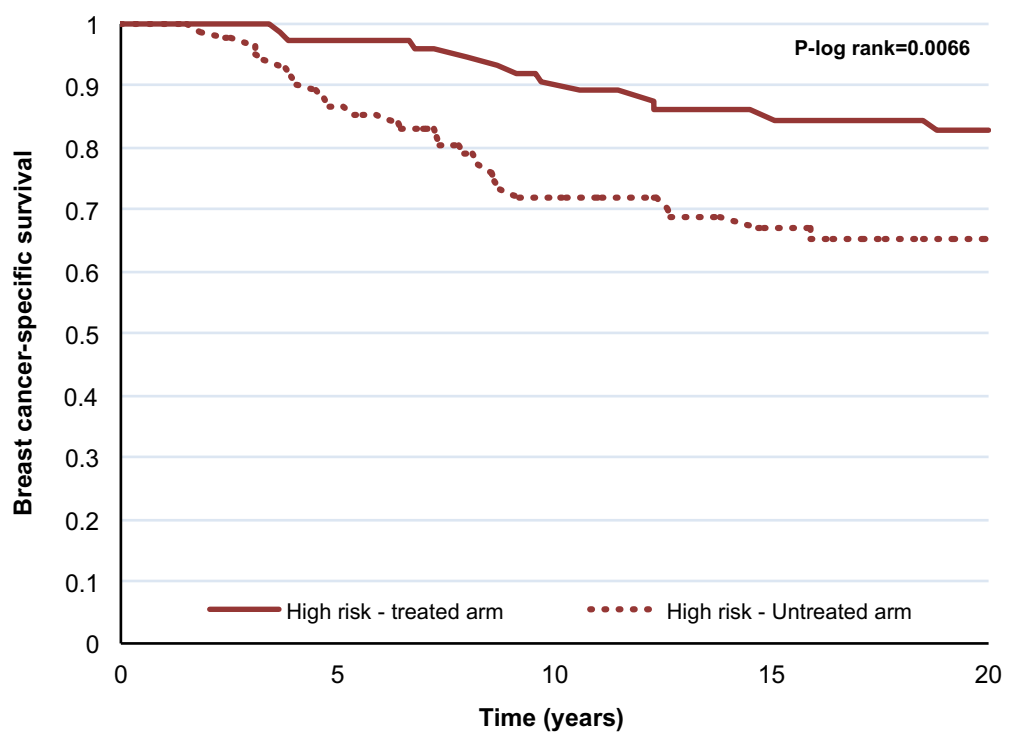

\begin{tabular}{|l|l|l|l|l|l|}
\multicolumn{1}{|c}{ Numbers at risk } & $\mathbf{0}$ & $\mathbf{5}$ & $\mathbf{1 0}$ & $\mathbf{1 5}$ & \multicolumn{2}{c|}{$\mathbf{2 0}$} \\
\hline High risk - Treated & 82 & 74 & 64 & 55 & 44 \\
\hline High risk - Untreated & 85 & 71 & 51 & 40 & 31 \\
\hline
\end{tabular}

B

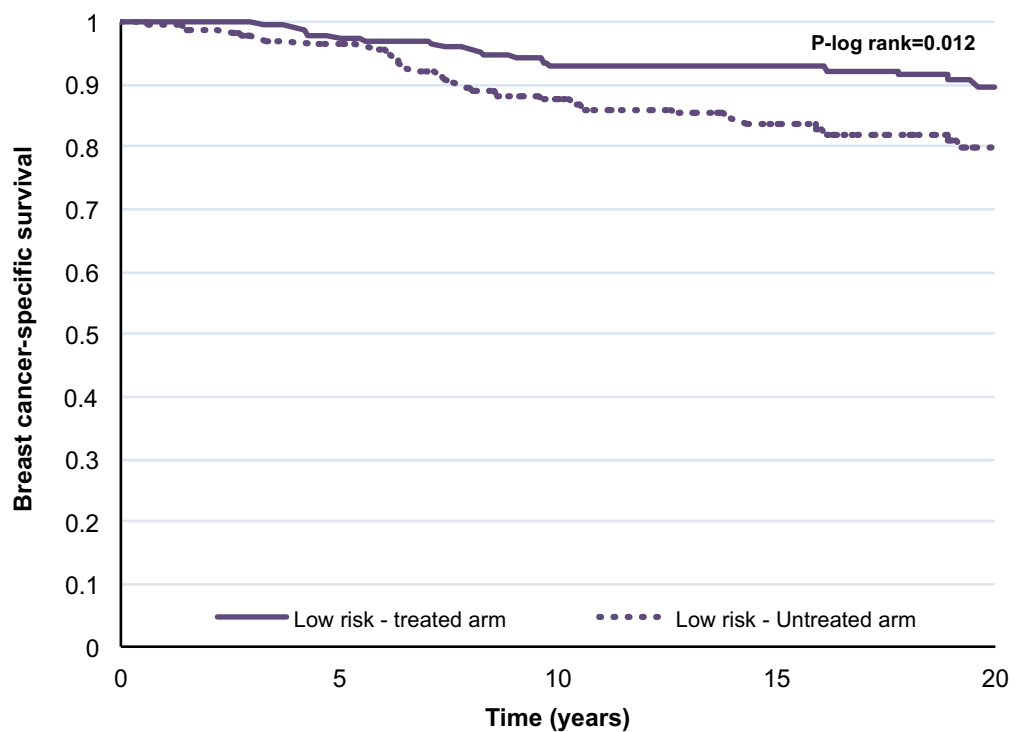

\begin{tabular}{|l|l|l|l|l|l|}
\multicolumn{1}{l}{ Numbers at risk } & $\mathbf{0}$ & $\mathbf{5}$ & $\mathbf{1 0}$ & $\mathbf{2 0}$ \\
\hline Low risk - Treated & 199 & 186 & 162 & 129 & 90 \\
\hline Low risk - Untreated & 172 & 157 & 125 & 104 & 69 \\
\hline
\end{tabular}

specific survival by STO-3 trial arm (tamoxifen-treated versus untreated) was seen within the high- as well as the low-risk group (70-gene high risk, tamoxifen yes/no: logrank $p=0.0066,70$-gene low risk, tamoxifen yes/no: logrank $p=0.012$ ). The benefit of tamoxifen was further investigated in the earlier defined ultralow-risk group of breast cancer patients with indolent disease and extremely good outcome [19]. No statistically significant difference in long-term survival was seen (Fig. 2, log-rank $p=0.39$ ) in the ultralow-risk group for patients receiving tamoxifen therapy or not.

In order to compare results to previous studies that evaluated endocrine treatment benefit in prognostic highand low-risk subgroups up to 10 year after diagnosis, we also performed a 10-year analysis of distant metastasis-free survival by the 70-gene risk classification using KaplanMeier analysis (four groups: low risk/treated arm, low risk/ untreated arm, high risk/treated arm, and high risk/ 
Fig. 2 Kaplan-Meier analysis of breast cancer-specific survival by 70 -gene ultralow risk and trial arm. The $p$ value is based on the log-rank test, and numbers at risk are shown underneath the graph

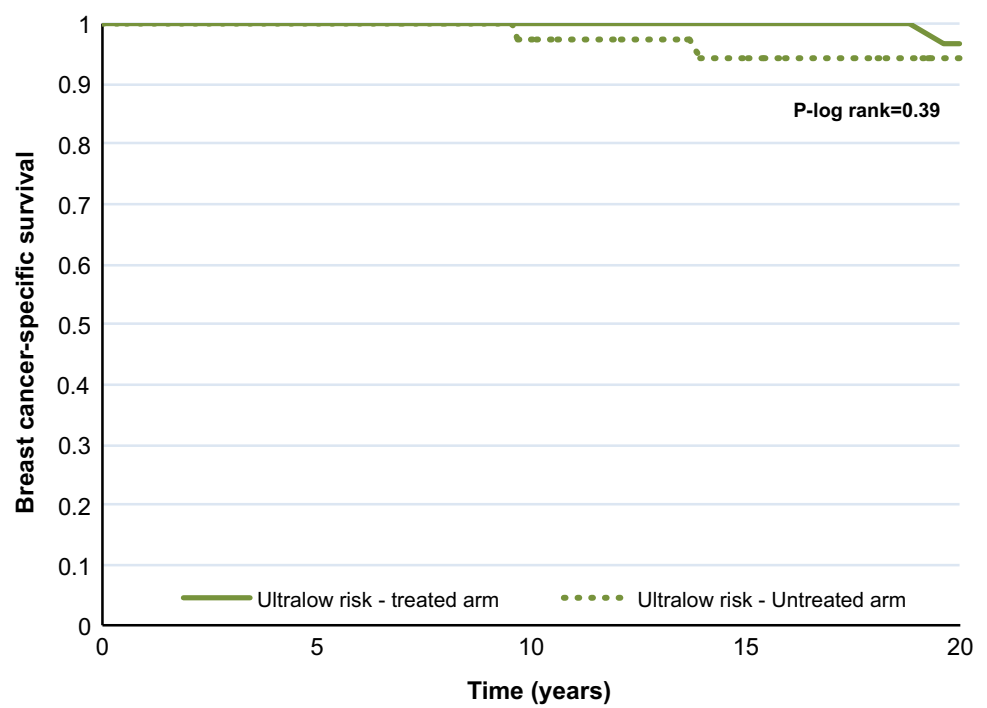

\begin{tabular}{|l|l|l|l|l|l|} 
Numbers at risk & $\mathbf{0}$ & $\mathbf{5}$ & $\mathbf{1 0}$ & $\mathbf{1 5}$ & $\mathbf{2 0}$ \\
\hline Ultralow risk - Treated & 54 & 52 & 48 & 39 & 28 \\
\hline Ultralow risk - Untreated & 44 & 43 & 36 & 30 & 19 \\
\hline
\end{tabular}

untreated arm). A statistically significant difference in 10-year distant metastasis-free survival was seen (Fig. 2S $\log$-rank, $p<0.0001$ ). For the 70-gene low-risk group, the 10 -year distant metastasis-free survival with and without tamoxifen treatment was 93\% (95\% CI 88-96\%) and $83 \%$ (95\% CI 76-88\%), respectively. For the 70-gene high-risk group, the 10-year distant metastasis-free survival with and without tamoxifen treatment was $85 \%$ (95\% CI 75-91\%) and $70 \%$ (95\% CI 58-79\%), respectively.

\section{Multivariable survival analysis}

Multivariable analysis of long-term (20 years) breast cancer-specific survival by STO-3 trial arm in the 70-gene high-risk and the low-risk subgroups was performed using Cox proportional hazard modeling adjusting for classical patient and tumor characteristics (age and calendar period of diagnosis, progesterone receptor status, HER2 status, Ki-67 status, tumor grade, and tumor size). Interestingly, both patients classified as 70-gene high, as well as low risk notably benefited from tamoxifen treatment (Table 2) after adjusting for clinico-pathological factors. Patients with high-risk tumors that were in the tamoxifen trial arm had a significantly reduced risk of dying from breast cancer, relative risk reduction of $58 \%$, as compared to patients in the untreated arm [Hazard ratio (HR), 0.42; 95\% CI 0.21-0.86]. Low-risk patients that were treated with tamoxifen had a significantly reduced risk (relative risk reduction of 54\%) of dying from breast cancer [Hazard ratio (HR), 0.46 ; 95\% CI $0.25-0.85]$.
Finally, time-dependent multivariable analyses were also performed $1,5,10,15$, and 20 years after breast cancer diagnosis using flexible parametric survival models to estimate hazard ratios. For both low- and high-risk tumors, patients had a significant benefit of tamoxifen treatment up to 10 years after diagnosis but less benefit after (Table 3).

\section{Discussion}

In this study, we had the rare opportunity to observe the long-term (20-year) impact of tamoxifen therapy versus not as the sole adjuvant therapy in women whose tumors were retroactively molecularly classified as either 70-gene high or low risk. The results demonstrate that there is a significant and comparable risk reduction benefit from tamoxifen in both groups, and that the survival benefit after 2 years of tamoxifen use (for one-third of patients in the tamoxifen arm after 5 years) continues for well over 10 years. These findings also confirm what has previously been shown, that the 70-gene classification is prognostic, and that women with low risk versus high risk have higher survival independent of treatment. In this study, adjuvant tamoxifen appeared to reduce risk of death by $50 \%$, regardless of 70-gene high- or low-risk biology.

It has been shown that breast cancer stage and grade do not appreciably affect the proportional risk reduction benefit from 5 years of adjuvant tamoxifen therapy [3]; this study, however, represents the first comparative analysis of 
Table 2 Risk of long-term breast cancer-specific death (20 year) by 70-gene classification and trial arm in ER-positive breast cancer

\begin{tabular}{|c|c|c|c|c|c|}
\hline \multirow{2}{*}{$\begin{array}{l}\text { STO-3 trial } \\
\text { Patients included }\end{array}$} & \multicolumn{2}{|c|}{ STO-3 trial arm } & \multirow[t]{2}{*}{$\begin{array}{l}\text { Breast cancer-specific } \\
\text { deaths }^{\mathrm{a}}\end{array}$} & \multicolumn{2}{|c|}{$\begin{array}{l}\text { Breast cancer-specific } \\
\text { survival }^{\text {a }}\end{array}$} \\
\hline & Trial arm & Number & & $\mathrm{HR}(95 \% \mathrm{CI})$ & $\begin{array}{l}p \text { value } \\
\left(\chi^{2}\right)\end{array}$ \\
\hline \multicolumn{6}{|l|}{ 70-gene High risk } \\
\hline \multirow[t]{2}{*}{$\begin{array}{l}\text { Adjusting for classical patient and tumor } \\
\text { characteristics }^{£}\end{array}$} & Treated $\operatorname{arm}^{\mathrm{b}}$ & 82 & 12 & $\begin{array}{l}0.42 \\
\quad(0.21-0.86)\end{array}$ & $\begin{array}{r}0.018 \\
(5.62)\end{array}$ \\
\hline & $\begin{array}{l}\text { Untreated } \\
\text { arm }\end{array}$ & 85 & 26 & 1.0 ref. & \\
\hline \multicolumn{6}{|l|}{ 70-gene Low risk } \\
\hline \multirow[t]{2}{*}{$\begin{array}{l}\text { Adjusting for classical patient and tumor } \\
\text { characteristics }^{£}\end{array}$} & Treated $\mathrm{arm}^{\mathrm{b}}$ & 199 & 17 & $\begin{array}{l}0.46 \\
\quad(0.25-0.85)\end{array}$ & $\begin{array}{r}0.013 \\
(6.15)\end{array}$ \\
\hline & $\begin{array}{l}\text { Untreated } \\
\text { arm }\end{array}$ & 172 & 29 & 1.0 ref. & \\
\hline
\end{tabular}

Hazard rates in bold indicate statistically significant values

a 20-year breast cancer-specific survival

b Modeled by multivariable proportional hazard (Cox) analyses adjusting for age and calendar period of diagnosis, progesterone receptor status, HER2 status, Ki-67 status, tumor grade, and tumor size

Table 3 Time-varying analysis of the long-term risk for breast cancer-specific death (20 years) by 70 -gene classification and trial arm in ERpositive breast cancer

\begin{tabular}{|c|c|c|c|c|c|}
\hline \multirow{2}{*}{$\begin{array}{l}\text { STO-3 trial } \\
\text { Patients included }\end{array}$} & \multicolumn{2}{|l|}{ STO-3 trial arm } & \multirow[t]{2}{*}{ Breast cancer-specific deaths ${ }^{\mathrm{a}}$} & \multirow[t]{2}{*}{ Years since diagnosis } & \multirow[t]{2}{*}{ HR $(95 \% \mathrm{CI})$} \\
\hline & Trial arm & Number & & & \\
\hline \multirow[t]{6}{*}{ 70-gene High risk ${ }^{\mathrm{b}}$} & Treated arm & 82 & 12 & 1 & $0.20(0.05-0.74)$ \\
\hline & & & & 5 & $0.37(0.21-0.67)$ \\
\hline & & & & 10 & $0.55(0.33-0.90)$ \\
\hline & & & & 15 & $0.81(0.33-1.99)$ \\
\hline & & & & 20 & $1.00(0.31-3.28)$ \\
\hline & Untreated arm & 85 & 26 & 1.0 ref. & \\
\hline \multirow[t]{6}{*}{ 70-gene Low risk ${ }^{\mathrm{b}}$} & Treated arm & 199 & 17 & 1 & $0.20(0.05-0.75)$ \\
\hline & & & & 5 & $0.37(0.20-0.67)$ \\
\hline & & & & 10 & $0.53(0.3-0.87)$ \\
\hline & & & & 15 & $0.75(0.35-1.62)$ \\
\hline & & & & 20 & $0.90(0.33-2.84)$ \\
\hline & Untreated arm & 172 & 29 & $1.0 \mathrm{ref}$ & \\
\hline
\end{tabular}

Hazard rates in bold indicate statistically significant values

a 20 -year breast cancer-specific survival

b Modeled by flexible parametric survival analysis adjusting for age and calendar period of diagnosis, progesterone receptor status, HER2 status, Ki-67 status, tumor grade, and tumor size

adjuvant tamoxifen's risk reduction benefit based on a priori molecularly defined risk categorization, specifically the 70-gene prognosis signature low- and high-risk subgroups. These STO-3 outcome data also serve to remind us about the natural history of HR-positive breast cancer and illustrate the very long tail of death from breast cancer. Even, those women with low-risk tumor biology continue to have risk of death, and in fact their risk of death is higher after 5 years and this continues for at least 20 years. For those women with molecularly high risk (by the 70-gene classification), their recurrence risk also persists up to 20 years, but the bulk of the risk is experienced in the first 5 or (to lesser extent) 10 years. Interestingly, the benefit of adjuvant tamoxifen treatment shows the same proportional reduction of risk of death over time for both low- and highrisk groups. In contrast to this observation, for those STO-3 patients for whom we recently defined having a 70-gene indolent/ultralow-risk disease with an extremely low 
20-year risk of death [19], we show that BCSS benefit from adjuvant tamoxifen does not achieve clinical significance over a period of 20 years relative to untreated patients with ultralow-risk ER-positive breast cancer.

Most prognostic tools have been shown to determine risk of recurrence only out to 10 years in a uniformly endocrine-treated population, making these tools less suitable to evaluate their ability to identify which patients might and to what extent benefit from endocrine therapy [3]. This is the first time a prognostic tool shows a benefit of tamoxifen as the sole adjuvant therapy for molecularly identified high-risk patients, alongside the benefit in lowrisk patients. The reason that the 70-gene signature is able to identify this benefit across all risk groups is based on the fact that the signature was developed in an adjuvant treatment-naïve population, whereas other prognosis signatures like Oncotype and Endopredict have been developed on tamoxifen-treated population $[9,22]$. The benefit of tamoxifen is therefore difficult to discern [9]. Our analysis of distant metastasis-free survival at 10 years also allows direct comparison with previously published papers for patients having received endocrine treatment which results in comparable 10-year DMFS rates [9, 22, 23].

Interestingly, this paper also likely confirms the additive benefit of chemotherapy, as risk for recurrence is more substantial in the first 5 years in the high-risk group as compared to the low-risk group. Even if the relative benefit of tamoxifen risk reduction is the same, the absolute risk of recurrence is higher in the high-risk group, where chemotherapy is known to exert its optimal effect [24]. In the low-risk and particularly the ultralow-risk group, the absolute risk of recurrence is extremely low in the first 5 years, therefore making it unlikely that there would be benefit from chemotherapy, an observation recently made in the large prospective randomized MINDACT trial for breast cancers with 70-gene low-risk signature even within the clinical high-risk setting [10, 25].

In order to advance the science of personalized medicine, diagnostics need to help us determine who will benefit and when. Women with ultralow-risk profiles have almost no risk and therefore benefit little if at all even from endocrine treatment. Women with biologically high-risk disease benefit from tamoxifen, but have high early residual risk supporting a decision to intercede with chemotherapy. Women with low- but not ultralow-risk disease, as well as those with high-risk disease, would be very well served if we had a robust marker of sensitivity to endocrine therapy and chemotherapy, to understand their long-term risk to die from breast cancer. That way, we could find ways to intercede for this group of women, and, as well, to determine for whom endocrine therapy is not sufficient. In order to more rapidly advance the field, we have to be able to identify those women at diagnosis and specifically focus more targeted interventions. Establishing the long-term benefit of endocrine therapy in prognostic subclasses as described here contributes to our ability to guide the use of adjuvant therapy in breast cancer.

Acknowledgements We thank Neil E. Hubbard, Olanu Aina, Judith Walls, and Herlina Sugandha of UC Davis for help with slide staining and organization for the reader scoring and the ATHENA pathologists for help with the scoring of ER, PR, HER2, and Ki-67. Gene expression array experiments and 70-gene assessment were provided in kind by Agendia Inc, Irvine US. This work was supported by the California Breast Cancer Research Program BCRP award [Grant No.: 180B-0065, Predicting Breast Cancer Recurrence To Improve Care to LJE], the Breast Cancer Research Foundation [BCRF to LJV], the Swedish Research Council [Grant No. 521-2014-2057 to LSL], FORTE [Grant No. 2014-1962 to LSL], and Stiftelsen Gösta Miltons Donationsfond [The Gösta Milton Donation Fund to LSL] and Cancerföreningen i Stockholm [Stockholm Cancer Society to LSL].

Compliance with ethical standards The experiments and analyses comply with the current laws of the country in which they were performed.

Conflict of interest All authors (except LJV) have declared no relevant affiliations or financial involvement with any organization or entity with a financial interest in or financial conflict with the subject matter or materials discussed in the manuscript. LJV is part-time employee of Agendia N.V., the commercial entity that markets the 70-gene signature as MammaPrint. LJV is co-founder and shareholder of Agendia, and named inventor on the patent for the 70-gene signature used in this study. The 70-gene patent holder is the Netherlands Cancer Institute.

Open Access This article is distributed under the terms of the Creative Commons Attribution 4.0 International License (http://crea tivecommons.org/licenses/by/4.0/), which permits unrestricted use, distribution, and reproduction in any medium, provided you give appropriate credit to the original author(s) and the source, provide a link to the Creative Commons license, and indicate if changes were made.

\section{References}

1. Colleoni M, Sun Z, Price KN et al (2016) Annual hazard rates of recurrence for breast cancer during 24 years of follow-up: results from the international breast cancer study group trials I to V. J Clin Oncol 34:927-935

2. Early Breast Cancer Trialists' Collaborative Group, Davies C, Godwin J et al (2011) Relevance of breast cancer hormone receptors and other factors to the efficacy of adjuvant tamoxifen: patient-level meta-analysis of randomised trials. Lancet 378:771-784

3. Schiavon G, Smith IE (2014) Status of adjuvant endocrine therapy for breast cancer. Breast Cancer Res 16:206

4. Jirstrom K, Ryden L, Anagnostaki L et al (2005) Pathology parameters and adjuvant tamoxifen response in a randomized premenopausal breast cancer trial. J Clin Path 58:1135-1142

5. Sheppard VB, Faul LA, Luta G et al (2014) Frailty and adherence to adjuvant hormonal therapy in older women with breast cancer: CALGB protocol 369901. J Clin Oncol 32:2318-2327

6. Sorlie T, Perou CM, Tibshirani R et al (2001) Gene expression patters of breast carcinomas distinguish tumor subclasses with clinical implications. Proc Natl Acad Sci USA 98:10869-10874 
7. Curtis C, Shah SP, Chin SF et al (2012) The genomic and transcriptomic architecture of 2,000 breast tumours reveals novel subgroups. Nature 486:346-352

8. Sotiriou C, Pusztai L (2009) Gene-expression signatures in breast cancer. N Eng J Med 360:790-800

9. Paik S, Shak S, Tang G et al (2004) A multigene assay to predict recurrence of tamoxifen-treated, node-negative breast cancer. N Engl J Med 351:2817-2826

10. Cardoso F, van't Veer LJ, Bogaerts J et al (2016) 70-Gene signature as an aid to treatment decisions in early-stage breast cancer. N Engl J Med 375:717-729

11. van't Veer LJ, Dai H, van de Vijver MJ et al (2002) Gene expression profiling predicts clinical outcome of breast cancer. Nature 415:530-536

12. Jerevall PL, Ma XJ, Li $\mathrm{H}$ et al (2011) Prognostic utility of HOXB13:IL17BR and molecular grade index in early-stage breast cancer patients from the Stockholm trial. $\mathrm{Br} \mathrm{J}$ Cancer 104:1762-1769

13. McShane L, Altman D, Saurerbrei W et al (2006) Reporting recommendations for tumor marker prognostic studies (REMARK). Breast Cancer Res Treat 100:229-235

14. Larsson LG, Nystrom L, Wall S et al (1996) The Swedish randomised mammography screening trials: analysis of their effect on the breast cancer related excess mortality. J Med Screen 3:129-132

15. Rutqvist LE (1985) Validity of certified causes of death in breast carcinoma patients. Acta Radiol Oncol 24:385-390

16. Barlow L, Westergren K, Holmberg L, Talback M (2009) The completeness of the Swedish Cancer Register: a sample survey for year 1998. Acta Oncol 48:27-33

17. Beumer I, Witteveen A, Delahaye L et al (2016) Equivalence of MammaPrint array types in clinical trials and diagnostics. Breast Cancer Res Treat 156:279-287
18. Delahaye LJ, Drukker CA, Dreezen C et al (2017) A breast cancer gene signature for indolent disease. Breast Cancer Res Treat 164:461-466

19. Esserman LJ, Yau C, Thompson CK et al (2017) Use of molecular tools to identify patients with indolent breast cancers with ultralow risk over 2 decades. JAMA Oncol. doi:10.1001/jamaon col.2017.1261

20. Lambert PC, Royston P (2009) Further developments of flexible parametric models for survival analysis. Stata J 9:265-290

21. Royston P, Parmar MK (2002) Flexible parametric proportionalhazards and proportional-odds models for censored survival data, with application to prognostic modelling and estimation of treatment effects. Stat Med 21:2175-2197

22. Filipits M, Rudas M, Jakesz R et al (2011) A new molecular predictor of distant recurrence in ER-positive, HER2-negative breast cancer adds independent information to conventional clinical risk factors. Clin Cancer Research 17:6012-6020

23. Albain KS, Barlow WE, Shak S et al (2010) Prognostic and predictive value of the 21- gene recurrence score assay in postmenopausal women with node-positive, oestrogen-receptor-positive breast cancer on chemotherapy: a retrospective analysis of a randomised trial. Lancet Oncol 11:55-65

24. Early Breast Cancer Trialists' Collaborative Group (EBCTCG) (2005) Effects of chemotherapy and hormonal therapy for early breast cancer on recurrence and 15-year survival: an overview of the randomized trials. Lancet 365:1687-1717

25. Krop I, Ismaila N, Andre F et al (2017) Use of biomarkers to guide decisions on adjuvant systemic therapy for women with early-stage invasive breast cancer: American Society of Clinical oncology Clinical Practice Guideline Focused Update. J Clin Oncol. doi:10.1200/JCO.2017.74.0472 